The International Journal of Multimedia \& Its Applications (IJMA) Vol.4, No.6, December 2012

\title{
DENOISING OF MAGNETIC RESONANCE AND X-RAY IMAGES USING VARIANCE STABILIZATION AND PATCH BASED ALGORITHMS
}

\author{
V N Prudhvi Raj ${ }^{1}$ and Dr T Venkateswarlu ${ }^{2}$ \\ ${ }^{1}$ Associate Professor, VR Siddhartha Engineering College, Vijayawada, 520007, India \\ nagaprudhvi@yahoo.com \\ ${ }^{2}$ Professor, University College of Engineering, SV University, Tirupati, India
}

\begin{abstract}
Developments in Medical imaging systems which are providing the anatomical and physiological details of the patients made the diagnosis simple day by day. But every medical imaging modality suffers from some sort of noise. Noise in medical images will decrease the contrast in the image, due to this effect low contrast lesions may not be detected in the diagnostic phase. So the removal of noise from medical images is very important task. In this paper we are presenting the Denoising techniques developed for removing the poison noise from X-ray images due to low photon count and Rician noise from the MRI (magnetic resonance images). The Poisson and Rician noise are data dependent so they won't follow the Gaussian distribution most of the times. In our algorithm we are converting the Poisson and Rician noise distribution into Gaussian distribution using variance stabilization technique and then we used the patch based algorithms for denoising the images. The performance of the algorithms was evaluated using various image quality metrics such as PSNR (Peak signal to noise ratio), UQI (Universal Quality Index), SSIM (Structural similarity index) etc. The results proved that the Anscombe transform, Freeman \& Tukey transform with block matching $3 D$ algorithm is giving a better result.
\end{abstract}

\section{KEYWORDS}

Variance Stabilization Transform, Poisson Noise, Nonlocal Means, Block matching.

\section{INTRODUCTION}

Medical imaging became an integral part of medical diagnosis in present days. Various medical imaging modalities are developed for various applications since last few decades. These modalities are used to acquire the images of the anatomical structures within the body to be examined without opening the body. X-rays, Computed Tomography, Ultrasound, Magnetic resonance Imaging and Nuclear imaging are the popular modalities at present to diagnose the various diseases. However these modalities are suffering with a big problem called noise. Every modality is suffering from noise in image acquisition and transmission stage such as Quantum noise in X-rays and Nuclear imaging, speckle noise in ultrasound imaging, Rician noise in Magnetic resonance imaging etc. The noise present in the images will degrade the contrast of the image and creates problems in the diagnostic phase. So denoising is very important to remove the noise from these images [20].

The noise may be additive or multiplicative depending on the modality used for medical image acquisition. The noise due to electronic components in the acquisition hardware will be modeled with Gaussian noise which independent of data, the data dependent noise such as quantum noise in X-ray imaging is modeled with Poisson distribution, the speckle noise in ultrasound imaging is modeled with Rayleigh distribution and the noise in MRI is modeled with Rician distribution.

DOI : 10.5121/ijma.2012.4605 
The International Journal of Multimedia \& Its Applications (IJMA) Vol.4, No.6, December 2012

Here in this paper we are attempting to denoise the images corrupted with quantum noise in X-ray and Nuclear imaging and Rician noise in Magnetic Resonance Imaging [20, 12].

The mathematical modeling of degradation and restoration process is given as

$$
\begin{aligned}
& g(x, y)=f(x, y) * h(x, y)+\eta(x, y) \\
& G(u, v)=F(u, v) H(u, v)+N(u, v)
\end{aligned}
$$

Where $g(x, y)$ is the noisy and blurred observation, $H$ is the blurring kernel and $f(x, y)$ is the signal we are recovering. In the case of denoising problem the blurring kernel will be dropped and the degradation model will be given as

$$
\begin{aligned}
& g(x, y)=f(x, y)+\eta(x, y) \\
& G(u, v)=F(u, v)+N(u, v)
\end{aligned}
$$

In the case of multiplicative noise the model is given as

$$
g(x, y)=f(x, y) \cdot \eta(x, y)
$$

\section{MATHEMATICAL PROPERTIES OF NOISE}

\subsection{Poisson Noise}

The noise in X-ray imaging and Nuclear Imaging (PET, SPECT) is modeled with Poisson noise. $\mathrm{X}$-ray photons incident on a receptor surface in a random pattern. We cannot force them to be evenly distributed over the receptor surface. One area of the receptor surface may receive more photons than another area, even when both the areas are exposed to the same average x-ray intensity. In all medical imaging procedures using gamma or x-ray photons most of the image noise is produced by the random behaviour of the photons that are distributed within the image. This is generally designated quantum noise. Each individual photon is a quantum (specific quantity) of energy. It is the quantum structure of an x-ray beam that creates quantum noise [9].

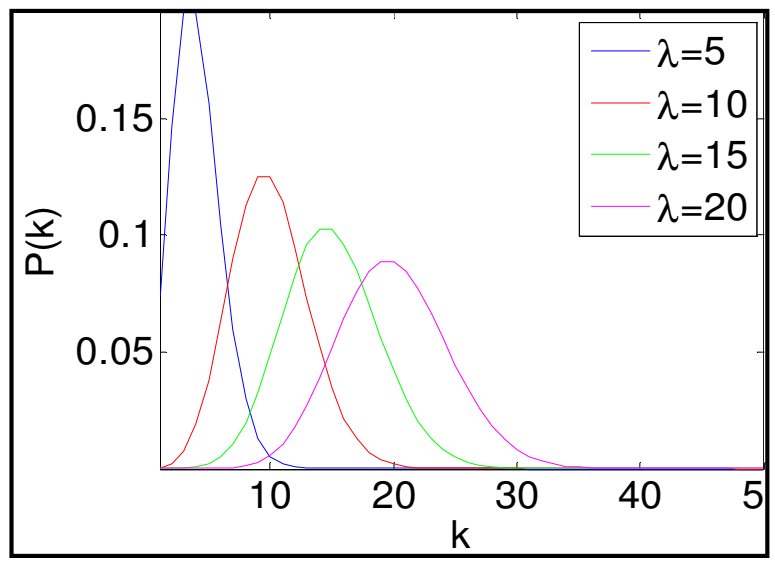

Figure 1: Poisson distribution for various values of lambda 
The International Journal of Multimedia \& Its Applications (IJMA) Vol.4, No.6, December 2012

A Poisson model assume that each pixel ' $x$ ' of an image ' $f(x)$ 'is drawn from a Poisson distribution of parameter ' $\lambda=f_{0}(x)$ 'where ' $f_{0}$ ' is the original image to recover. The Poisson density is given as

$$
P(f(x)=k)=\frac{\lambda^{k} e^{-\lambda}}{k !}
$$

The above figure gives the Poisson distribution for various values of ' $\lambda$ ' as the ' $\lambda$ 'increases the Poisson distribution turns towards the Gaussian distribution.

\subsection{Rician Noise}

Magnetic Resonance Imaging (MRI) is a non-invasive widely used modality in medical diagnosis such as cardiac related diseases and neurological disorders. The MRI imaging will suffer from low signal to noise ratio (SNR) or contrast to noise ratio (CNR) because of which the image analysis tasks such as segmentation, reconstruction and registration will become complicated. So the noise reduction in MR images is very important as a pre-processing task before going to image analysis and to improve the diagnostic quality of the images $[9,12]$.

Thermal noise is the major source of noise in MR imaging. The MR images are reconstructed from the raw data by applying the inverse Fourier transform to it. The signal component is present in both real and imaginary channels which are orthogonal to each other and are affected by additive white Gaussian noise. Hence the noise in the reconstructed date is complex white Gaussian noise. Normally the magnitude image of the reconstructed complex data is used for visual inspection. So the magnitude of the MR signal is the square root of the sum of the squares of the data present in real and imaginary channels, the noise is the square root of the two independent Gaussian variables. Hence the noise in MR images is no longer Gaussian.

Let ' $A$ ' be the pixel intensity in the absence of noise and ' $M$ 'be the observed or measured pixel intensity. In the presence of noise the probability distribution for ' $M$ ' is given as

$$
P_{M}(M)=\frac{\frac{M}{\sigma^{2}} e^{-\left(M^{2}+A^{2}\right)}}{2 \sigma^{2}} I_{0}\left(\frac{A \cdot M}{\sigma^{2}}\right)
$$

Where ' $\sigma$ 'denotes the standard deviation of the Gaussian noise in the real and imaginary images which is considered as equal here and ' $I_{o}{ }^{\prime}$ 'is the modified zeroth order of Bessel function of the first kind. This is called as Rice density. For small values of SNR $(A / \sigma \leq 1)$ the rice distribution is far from being Gaussian and from ratios as small as $A / \sigma=3$ it starts to move towards the Gaussian distribution.

In the image regions where signal content is much less (approximately zero i.e. $A=0$ ) only noise is present then the above equation is reduces to

$$
P_{M}(M)=\frac{\frac{M}{\sigma^{2}} e^{-\left(M^{2}\right)}}{2 \sigma^{2}}
$$

This is well known as Rayleigh distribution. This distribution governs the noise in image regions where no NMR signal and only noise is present. The mean and variance of this distribution is given as 
The International Journal of Multimedia \& Its Applications (IJMA) Vol.4, No.6, December 2012

$$
M=\sigma \sqrt{\frac{\pi}{2}} \text { and } \sigma_{M}^{2}=\left(2-\frac{\pi}{2}\right) \sigma^{2}
$$

These relations are useful in the estimation of the true noise power.
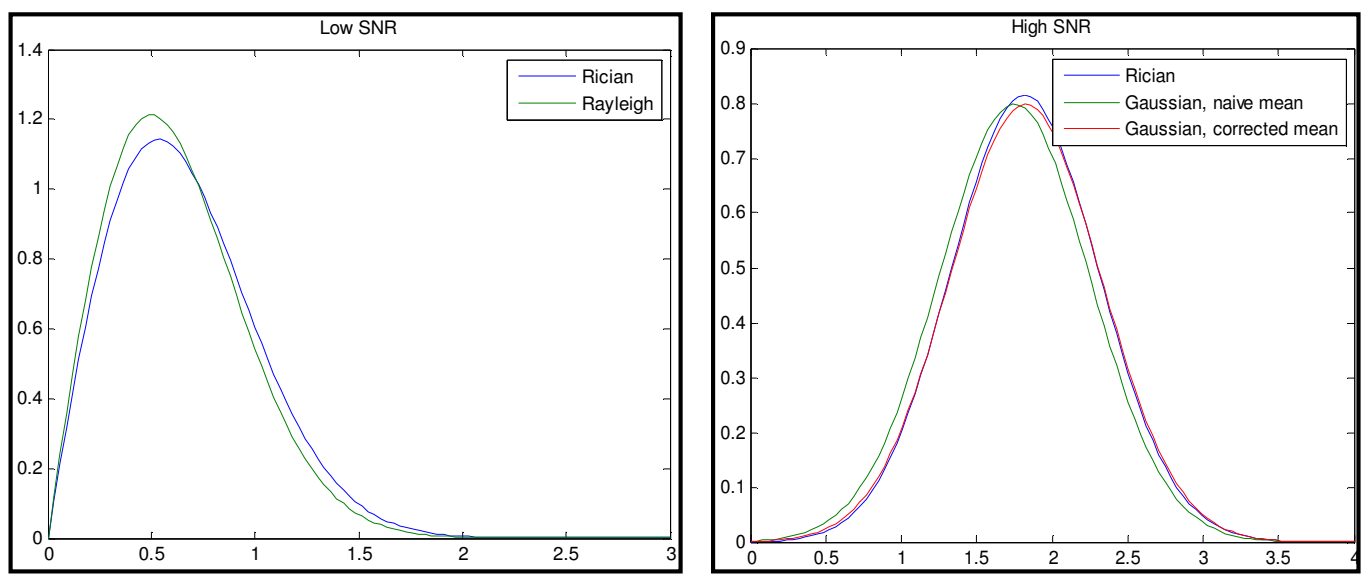

Figure 2: Rician noise (Low SNR, High SNR) distributions

When the SNR is large then

$$
P_{M}(M) \approx \frac{1}{\sqrt{2 \pi \sigma^{2}}} e^{\frac{-1\left(M-\sqrt{A^{2}}+\sigma^{2}\right)^{2}}{2 \sigma^{2}}}
$$

From the above equations we can say that for image regions where large signal intensities are present the noise distribution will be considered as a Gaussian distribution with mean $\sqrt{A^{2}+\sigma^{2}}$ and variance $\sigma^{2}$.

\section{Proposed METHOD}

A numerous algorithms are developed by the scientists and engineers to remove the noise in the natural and medical images since 1970. These denoising methods are originating from various disciplines such as algebra, probability \& statistics, linear and nonlinear filtering, partial differential equations and multiresolution analysis etc. At first the denoising methods are based on pixel by pixel transformation later the neighborhood filtering was developed to remove the noise. However these methods will introduce some artifacts and blurring while removing the noise from the images. The important features in the image such as edges and texture are going to be damaged in the process of noise removal.

To preserve the edges and textural information in the image many denoising methods were developed such as anisotropic diffusion, Laplacian pyramids, steerable pyramids, wavelet transforms, principle component analysis based methods, dictionary based approaches etc. The anisotropic diffusion will preserve the edges but loose the texture information. The multiresolution based approaches are sensitive to the high frequency discontinuities but fails to detect the spatial changes in the low frequencies properly. The dictionary based approaches are very time consuming and computational cost is very high to implement in real time. 
The International Journal of Multimedia \& Its Applications (IJMA) Vol.4, No.6, December 2012

In this paper we developed the algorithms combining the advantages of variance stabilization transforms and the famous patch based algorithms (Nonlocal means and BM3D) to recover the medical images corrupted with Quantum noise (X-ray, CT, and Nuclear Imaging) and the Rician noise (Magnetic resonance imaging). The block diagram of the proposed architecture is given below. First the noisy image is processed by the variance stabilization transform to have the distribution with constant variance (Poisson and Rician distributions are converted into Gaussian distribution). Then one of the patch based technique is applied on the stabilized data to remove the noise and then the processed data is converted back by the inverse variance stabilization transform to get the denoised image. Each block of the architecture is explained in the following sections $[21,27]$.

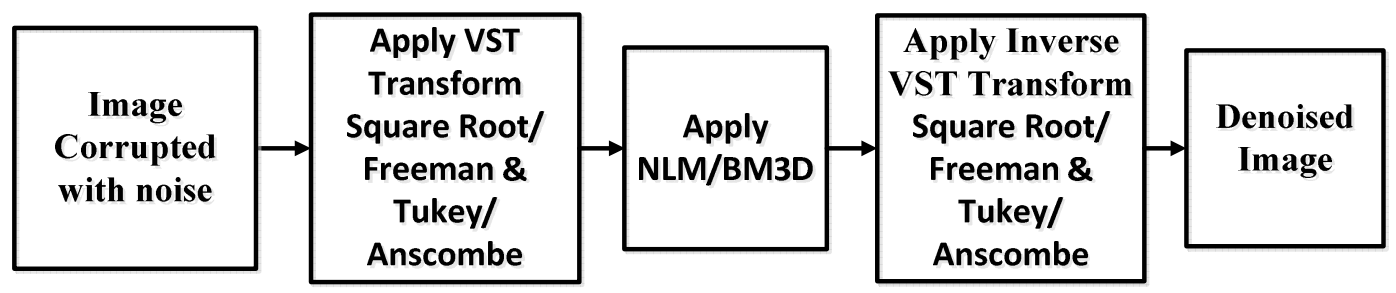

Figure 3: Proposed Denoising System Architecture

\subsection{Variance Stabilization Transforms}

In statistics the variance stabilization transforms are used to convert the distributions with variable variance into distribution with constant variance. These transforms are useful to convert the Poisson distribution and Rician distributions into Gaussian distribution with constant variance. The noise in the X-ray imaging and nuclear medical imaging such as PET and SPECT is modeled with Poisson distribution because its variance depends on the mean value and this makes difficult the denoising task. Here in this work we want to use the variance stabilization transforms as a preprocessing stage for the denoising filter to make the Poisson and Rician distributions into Gaussian distribution with known variance and then we perform the denoising operation. After the preprocessing we will apply the inverse variance stabilization transform to get back the original distributions. In this work we are using three variance stabilization transforms and their inverses they are square root, Freeman \& Tukey and Anscombe transforms. The mathematical representation of the transforms is given below. 
The International Journal of Multimedia \& Its Applications (IJMA) Vol.4, No.6, December 2012

Table 1: Variance Stabilization transforms and their Inverses

\begin{tabular}{|c|c|c|}
\hline Transform & Forward & Inverse \\
\hline Square Root & $Z=\sqrt{x}$ & $I=Z^{2}$ \\
\hline $\begin{array}{c}\text { Freeman \& } \\
\text { Tukey }\end{array}$ & $Z=\sqrt{x+1}+\sqrt{x}$ & $I=\left(\frac{Z^{2}-1}{2 Z}\right)^{2}$ \\
\hline Anscombe & $Z=2 \sqrt{x+\frac{3}{8}}$ & Adjusred: $I=\left(\frac{Z}{2}\right)^{2}-\frac{1}{8}$ \\
& & unbiased: $\quad$ Interpolation of computed values \\
\hline
\end{tabular}

The direct inverse and Adjusted inverse will give unbiased estimates if photon count is significant. However they will give biased estimates for the low intensity data that is photon count is minimum, the unbiased inverse is used in such situations.

\subsection{Gaussian Filters}

After transforming the data into distributions with constant variance we will apply the Gaussian filtering on this data. In this work we are using two algorithms one is nonlocal means algorithm and the second one is BM3D (block matching 3D). Here in this section we will give the brief description of these two algorithms.

In the earlier works of image denoising there are two assumptions considered about the noisy image. The first assumption is that the noisy image consists of both low and high frequencies. The noise is treated as non-smooth because of the high frequencies contained in it. The original image only contains low frequencies is the second assumption. i.e. images do not contain fine detail. Both the assumptions lead to the damage of fine details such as edges and lines in the denoised images.

The Wiener and the Gaussian filtering approaches also make these assumptions. The filters attempt to denoise the noisy image by removing the higher frequencies from the image keeping the lower frequencies assuming that noise is present in high frequencies and most of the image information is in low frequencies. The assumption will not suitable for the images which may not be smooth. They can contain fine details and structures which have high frequencies. Because these filtering methods unable to differentiate between the higher frequencies of the original image and the noise, the high frequencies of the original image will be lost. This results in blurring. In addition, the low frequencies of the noise will still remain in the denoised image. Here in this work we are not considering the above assumptions and our methods depends on the self-similarity of the pixels in the image. 
The International Journal of Multimedia \& Its Applications (IJMA) Vol.4, No.6, December 2012

\subsubsection{Nonlocal Means algorithm}

The nonlocal means filtering is also considered as the extension of the fundamental neighborhood filter (Yaroslavsky filter) where the pixel intensity is decided by averaging the intensities of its neighborhood.

In this method the pixels which are having similar neighborhood are found and averaged in deciding the intensity of a pixel. Normally similarity between the pixels is found by observing their intensities, if the two pixels having the same intensity we can say that they are similar otherwise they are different.

Here in the nonlocal means algorithm the similarity is found by considering the neighborhoods of the pixels. Two pixels are similar when the neighborhoods of the two pixels are having same intensities. So we can define the neighborhood of pixel ' $i$ ' is a set of pixels ' $j$ ' whose neighborhoods are similar to the neighborhood of ' $i$ '.

The following figure shows the similarity windows in the image [29].
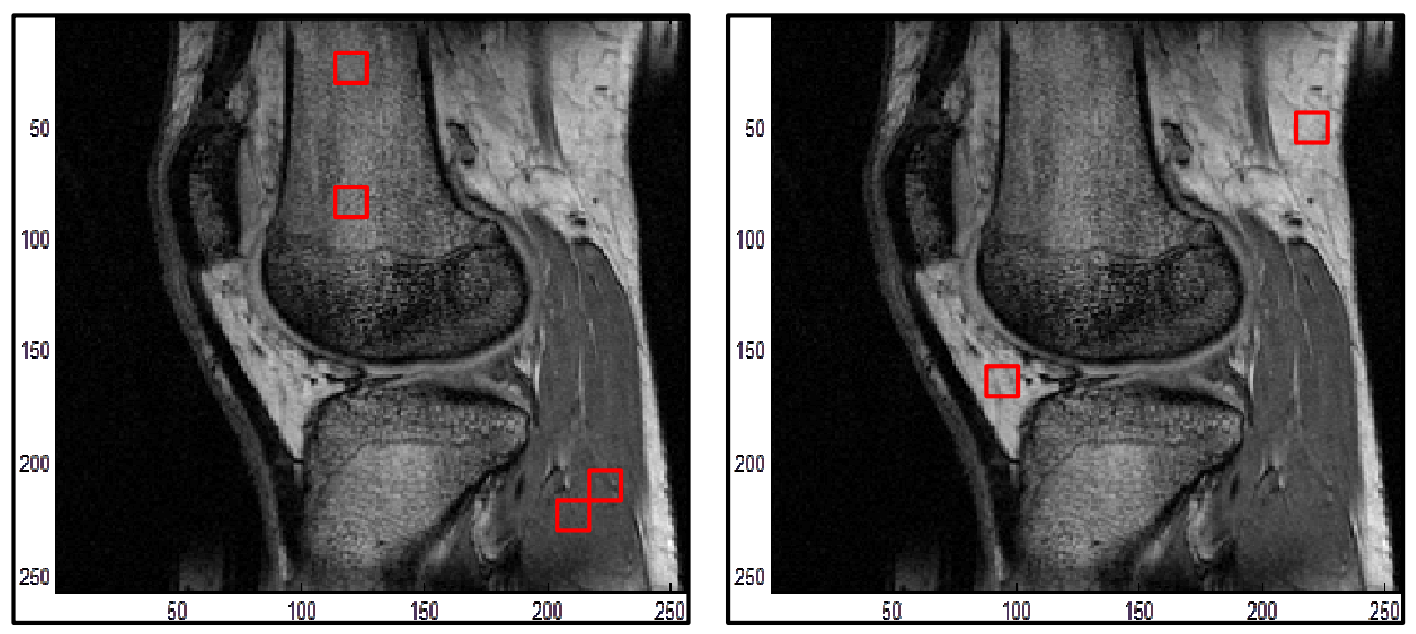

Figure 4: Self similarity in an Image (Fundamental to Patch based Algorithms)

Let ' $\Omega$ ' is the area of an image ' $I$ ', ' $x$ ' is the location inside ' $\Omega$ ', $u(x)$ and $v(x)$ are the clean and observed noisy image value at the location ' $x$ ' respectively. Then the nonlocal algorithm is given as follows

$$
N L[u](x)=\frac{1}{C(x)} \int_{\Omega} e^{-\frac{\left(G_{a} * v(x+.)-\left.v(y+.)\right|^{2}\right)(0)}{h^{2}}} v(y) d y
$$

Where $G_{a}$ a Gaussian is function with standard deviation ' $a$ ' and ' $h$ ' is a filtering parameter

$$
C(x)=\int_{\Omega} e^{-\frac{\left(G_{a} * v(x+.)-\left.v(y+.)\right|^{2}\right)(0)}{h^{2}}} d z
$$

In the discrete case the non-local means algorithm is given as

$$
N L[u](i)=\sum_{j \in I} w(i, j) v(j)
$$


The International Journal of Multimedia \& Its Applications (IJMA) Vol.4, No.6, December 2012

Where the weight $w(i, j)$ depends on the distance between observed gray level vectors at points ' $i$ 'and ' $j$ '. The distance can be calculated as

$$
d=\left\|v\left(N_{i}\right)-v\left(N_{j}\right)\right\|_{2, a}^{2}
$$

$N_{i}$ is the neighborhood of the pixel ' $i$ ' which is the squared window around the pixel ' $i$ '. Every pixel in the image will have their own neighborhood. The size of the neighborhood can be varied depending on the application. Now we can define ' $N S_{i}$ ' the neighborhood system of a pixel ' $i$ '. It is a set of similar neighborhoods to the neighborhoods of ' $i$ ' $\left(N_{i}\right)$. The similarity between the neighborhoods is calculated by calculating the difference in the intensity gray levels in the neighborhoods as shown in the above equation. The pixels ' $i$ ' and ' $j$ ' are said to be similar if $v\left(N_{i}\right)$ is similar to $v\left(N_{j}\right)$.

The weight can be defined as

$$
\begin{gathered}
w(i, j)=\frac{1}{z(i)} e^{-\frac{\left\|v\left(N_{i}\right)-v\left(N_{j}\right)\right\|_{2, a}^{2}}{h^{2}}} \\
\text { Where } z(i)=\sum_{j} e^{-\frac{\left\|v\left(N_{i}\right)-v\left(N_{j}\right)\right\|_{2, a}^{2}}{h^{2}}}
\end{gathered}
$$

\subsubsection{BM3D}

The BM3D algorithm is based on non-local image modelling and frequency domain filtering. The algorithm is developed based on enhanced sparse representation of the image. This can be achieved by the following steps. First the image is divided into number of $2 \mathrm{D}$ fragments. Then the similar 2D fragments are grouped together to form 3D stack. Now the collaborative filtering is performed to achieve the sparsity. And finally the filtered fragments are returned back to their original positions. The following figure gives the block diagram of the system [30].

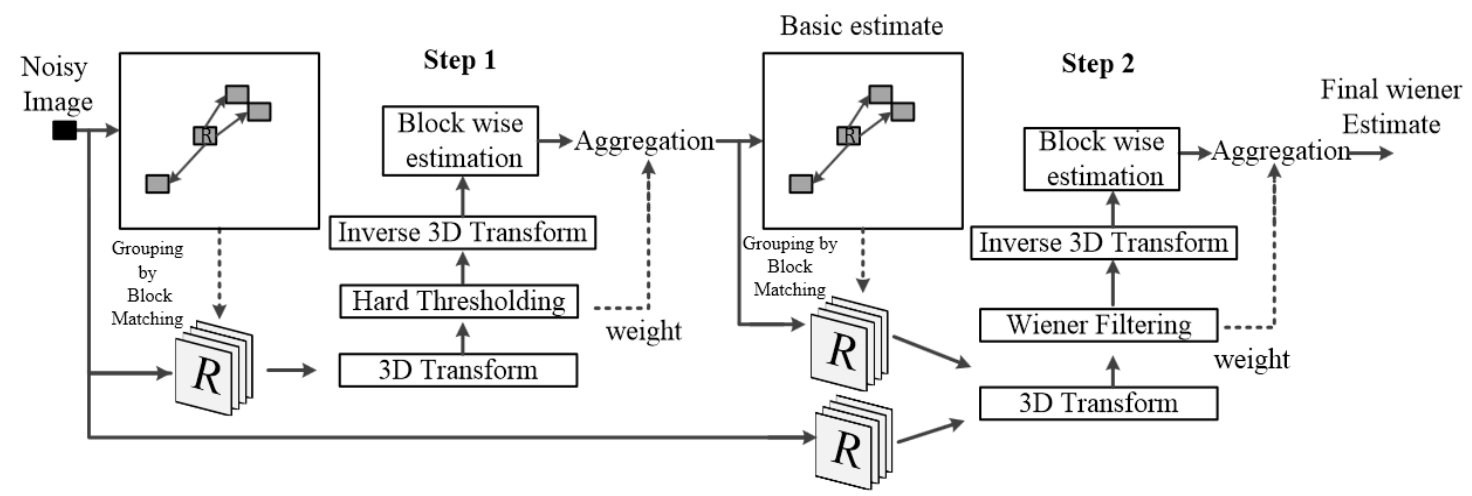

Figure 5: Block diagram of BM3D system used in our algorithm

The algorithm is divided into two stages. Basic estimation and Final estimation. In the basic estimation stage the groups are pre-filtered and this basic estimate is jointly used with noisy image in stage 2 to give the final estimate. The algorithm will proceed as follows. In both the 
The International Journal of Multimedia \& Its Applications (IJMA) Vol.4, No.6, December 2012

stages the estimation use similar steps except that in the first step filtering is carried by hard thresholding and in the second step filtering is done by wiener filtering.

\begin{tabular}{|ll|ll|}
\hline \multicolumn{1}{|c|}{ Basic Estimation } & \multicolumn{1}{c|}{ Final Estimation } \\
1. & Grouping. & 1. & Grouping (based on BE result) \\
2. & 3D Transform Application. & 2. & 3D Transform Application. \\
3. & Hard Thresholding. & 3. & Wiener filtering (using BE result). \\
4. & Inverse 3D Transform. & 4. & Inverse 3D Transform. \\
5. & Returned estimated blocks to their & 5. & Returned estimated blocks to their \\
& position & position. \\
6. & Aggregation & 6. & Aggregation. \\
\hline
\end{tabular}

\subsubsection{Grouping}

The first step in algorithm is to divide the image into number of fragments by defining a square neighborhood around each pixel. The groups are formed by performing block matching with reference to a reference frame to find the similar fragments at various spatial locations in the image. These $2 \mathrm{D}$ fragments are stacked as a $3 \mathrm{D}$ array.

The block matching method measures the difference between the fragments with reference one and if the difference is less than certain threshold the fragment is kept in the group. Let ' $N_{i}$ ' be the neighborhood around ' $i$ '(Reference fragment), ' $G N_{i}$ 'be the similarity group for this fragment, ' $T h$ ' is a certain threshold then the fragment ' $N_{j}$ ' belongs to the above group if it satisfies the following condition

$$
\text { If difference }\left(N_{i}, N_{j}\right)<T h \rightarrow N_{j} \in G N_{j}
$$

For grouping the similar blocks we may use the clustering algorithms but they will create the disjoint sets. Where the block matching algorithm one fragment may become member in more than one group. That is the algorithm produces disjoint sets.

\subsubsection{Similarity measurement}

Basic Estimation: In basic estimation the similarity is measured by applying a 2D forward transform on both the fragments and hard thresholding is performed on them. Now the difference between the spectral coefficients of both the fragments is measured. If the distance is smaller than certain threshold then the fragment is similar otherwise they are different from each other.

$$
D\left(N_{i}, N_{j}\right)=\frac{\left\|Y^{\prime}\left(T_{2 D}\left(N_{i}\right)\right)-Y^{\prime}\left(T_{2 D}\left(N_{j}\right)\right)\right\|_{2}^{2}}{\left(M_{1}\right)^{2}}
$$

$Y^{\prime}$ is the hard thresholding operator, $T_{2 D}$ is the $2 \mathrm{D}$ Linear Transform and $M_{1}$ is the fragment size in the first stage.

Final Estimation: The pre-filtered image from the basic estimation is used in forming the groups of second stage. The coordinates of the pre-filtered image is used to make the groups in the noisy image. The normalized Euclidean distance at this stage is given as 
The International Journal of Multimedia \& Its Applications (IJMA) Vol.4, No.6, December 2012

$$
D\left(P N_{i}, P N_{j}\right)=\frac{\left\|P N_{i}-P N_{j}\right\|_{2}^{2}}{\left(M_{2}\right)^{2}}
$$

Where $P N_{i}$ and $P N_{j}$ are the pre-filtered fragments, $M_{2}$ is the size of the fragment in second stage. Based on this new distances and new threshold new groups are defined.

Collaborative Filtering: The collaborative filtering consists of three steps. They are forward 3D transform, Filtering and Inverse 3D transform. The groups are characterized by intra-fragment correlation (appears between the pixels of each grouped fragment) and inter-fragment correlation (appears between the corresponding pixels of different fragments). The 3D transform can take advantage of these correlations and will produce the sparse representation of the true signal in the group. This sparsity will improve the effectiveness of shrinkage step in removing the noise and preserving the features of the image.

When a group of ' $X$ ' fragments are taken the collaborative filtering will produce ' $X$ ' estimates one for each fragment. Every fragment will participate in estimating the every other fragment so all the fragments are participating in the estimation process it is called collaborative filtering.

\section{Basic Estimation:}

In this stage the collaborative filtering is given as below

$$
P N G_{i}=T_{3 D}^{-1}\left(Y\left(T_{3 D}\left(G N_{i}\right)\right)\right)
$$

Where ' $Y$ ' is the hard thresholding operator, ' $T_{3 D}$ ' is the $3 \mathrm{D}$ linear transform, ' $G N_{i}$ ' is the group of fragments to be processed and ' $P N G_{i}$ ' is the output of the collaborative filtering. The $3 \mathrm{D}$ transform is implemented by performing the $2 \mathrm{D}$ transform on the fragments and $1 \mathrm{D}$ transform along the third dimension of the group. In this work biorthogonal splines are used in 2D transformation and Haar basis is used in 1D transform.

\section{Final Estimation:}

In this stage we have two groups one from noisy image and the other is pre-filtered image from basic estimation. This stage will use the wiener filtering. The coefficients of the wiener filter are computed from the pre-filtered image performing 3D transform on it as given below

$$
W_{C_{i}}=\frac{\left|T_{3 D}\left(P N G_{i}\right)\right|^{2}}{\left|T_{3 D}\left(P N G_{i}\right)\right|^{2}+\sigma^{2}}
$$

Now the collaborative filtering on the noisy fragments are carried out by multiplying the 3D transformed coefficients of noisy fragments with the wiener filter coefficients and taking the inverse 3D transform. This process will give us the final estimate.

$$
F N G_{i}=T_{3 D}^{-1}\left(W_{C_{i}} T_{3 D}\left(N G_{i}\right)\right)
$$

Where ' $W_{C_{i}}$ 'are the empirical wiener shrinkage coefficients, $T_{3 D}$ is a forward $3 \mathrm{D}$ linear transform, ' $N G_{i}{ }^{\prime}$ is the fragment group from noisy image and ' $F N G_{i}{ }^{\prime}$ is the final estimate. Here 
The International Journal of Multimedia \& Its Applications (IJMA) Vol.4, No.6, December 2012

in this stage the 2D transform is DCT (Discrete cosine transform) and 1D transform is Haar Transform.

\subsubsection{Aggregation:}

This is very important step in the algorithm. As the algorithm is not having disjoint sets in grouping the fragments may present more than one group. Because of this the fragments will get more than one estimation depending upon their participation. For example if a fragment is participating in three groups it will get three estimations one from each group after performing the collaborative filtering.

The aggregation in basic and final steps are performed by weighted averaging. The weights are computed based on the variance of the estimation i.e the weights are inversely related with the variance. If a fragment in having high variance it is considered as noisier and the weight associated with that frame is minimum. The weights are calculated as follows

\section{Basic estimation:}

$$
w_{B E}=\left\{\begin{array}{cl}
\frac{1}{\sigma^{2} N_{H T C}} & \text { if } \mathrm{N}_{H T C} \geq 1 \\
1 & \text { otherwise }
\end{array}\right.
$$

Where $N_{H T C}$ is the number of non-zero coefficients after hard thresholding

\section{Final estimation:}

$$
w_{F E}=\frac{1}{\sigma^{2}\left\|W_{C_{i}}\right\|_{2}^{2}}
$$

Where ' $W_{C_{i}}$ ' are the wiener filter coefficients.

Finally the weighted averaging is computed and each fragment is returned its original position.

\section{Evaluation CRiteria For DenOISING AlgorithmS}

To evaluate the quality of the image processing algorithms there are several metrics proposed in the literature. The metrics are classified as pixel difference based measures, correlation based measures, edge based measures, spectral distance measures, context based measures and Human visual system based measures. Here we are comparing our denoising algorithms using a group of metrics drawn from the above class and performance of the algorithms was observed.

\subsection{Pixel difference based measures}

\subsubsection{Minkowski metrics}

The $L_{\gamma}$ norm of the dissimilarity of two images can be calculated by calculating the minkowski average of the pixel differences spatially and then chromatically as given below

$$
\varepsilon^{\gamma}=\frac{1}{K} \sum_{k=1}^{K}\left\{\frac{1}{M N} \sum_{x=0}^{M-1} \sum_{x, y=0}^{N-1}\left|f_{k}(x, y)-\hat{f}_{k}(x, y)\right|^{\gamma}\right\}^{\frac{1}{\gamma}}
$$

Where $f(x, y)$ is the reference image, $\hat{f}(x, y)$ is the estimated image of $f(x, y)$ by our denoising algorithm with the input $g(x, y)$ which is a noisy version of $f(x, y)$. 
The International Journal of Multimedia \& Its Applications (IJMA) Vol.4, No.6, December 2012

For $\gamma=1$ we obtain the absolute difference (AD), for $\gamma=2$ we will obtain the mean square error (MSE). Along with these two measures we are calculating minkowski measures for $\gamma=3$ and $\gamma=4$ in this paper to observe the performance of our algorithms.

\subsubsection{PSNR (Peak Signal to Noise Ratio)}

PSNR is the peak signal-to-noise ratio in decibels (dB). The PSNR is only meaningful for data encoded in terms of bits per sample, or bits per pixel. For example, an image with 8 bits per pixel contains integers from 0 to 255 .

$$
P S N R=20 \log _{10}\left(\frac{2^{B}-1}{\sqrt{M S E}}\right)
$$

Where B represents bits per sample and MSE (Mean Squared error) is the mean square error between a signal $f(x, y)$ and an approximation $\hat{f}(x, y)$ is the squared norm of the difference divided by the number of elements in the signal.

$$
\begin{gathered}
M S E=\|f(x, y)-\hat{f}(x, y)\|^{2}=\frac{1}{M N} \sum_{x=0}^{M} \sum_{y=0}^{N}[f(x, y)-\hat{f}(x, y)]^{2} \\
R M S E=\sqrt{\frac{1}{M N} \sum_{x=0}^{M} \sum_{y=0}^{N}[f(x, y)-\hat{f}(x, y)]^{2}}
\end{gathered}
$$

MSE and RMSE measures the difference between the original and distorted sequences. PSNR measures the fidelity i.e how close a sequence is similar to an original one.

\subsubsection{Maximum Difference}

Maximum difference is defined as

$$
M D=\max (|f(x, y)-\hat{f}(x, y)|)
$$

The large value of maximum difference means denoised image is poor quality.

\subsubsection{Normalised Absolute Error (NAE)}

The large value of normalised absolute error means that denoised image is poor quality and is defined as

$$
N A E=\frac{\sum_{x=0}^{M-1} \sum_{y=0}^{N-1}|f(x, y)-\hat{f}(x, y)|}{\sum_{x=0}^{M-1} \sum_{y=0}^{N-1}|f(x, y)|}
$$

\subsubsection{Signal to Noise Ratio (SNR)}

Signal to noise ratio in an image is calculated as

$$
S N R=\frac{\mu}{\sigma}
$$


The International Journal of Multimedia \& Its Applications (IJMA) Vol.4, No.6, December 2012

Where $\mu$ is the average information in the signal and $\sigma$ is the standard deviation of the signal which represents the amount of noise present in the image. There is one more measure is there similar to the SNR it is signal to background ratio.

$$
S B R=\frac{\mu}{\sigma_{B G}}
$$

Subtract background from the image calculate standard deviation from it and finally compute the above ratio.

\subsection{Correlation based measures}

The correlation between two images can also be quantified interms of correlation function. These measures measure the similarity between the two images hence in this sense they are complementary to the difference based measures.

\subsubsection{Structural content}

For an $M \times N$ image the structural content is defined as

$$
S C=\frac{1}{K} \sum_{k=1}^{K} \frac{\sum_{x=0}^{M-1} \sum_{y=0}^{N-1} \sum_{y=0}^{N-1} \hat{f}_{k}(x, y)^{2}}{\sum_{y}}
$$

The large value of structural similarity means that denoised image is poor quality

\subsubsection{Normalised cross correlation measure (NK)}

The normalised cross correlation measure is defined as

$$
N K=\frac{1}{K} \sum_{k=1}^{K} \frac{\sum_{x=0}^{M-1} \sum_{y=0}^{N-1} f_{k}(x, y) \hat{f}_{k}(x, y)}{\sum_{x=0}^{M-1} \sum_{y=0}^{N-1} f_{k}(x, y)^{2}}
$$

\subsubsection{Czekanowski distance}

A metric useful to compare vectors with strictly positive components as in the case of images is given as

$$
C=\frac{1}{M N} \sum_{x=0}^{M-1} \sum_{y=0}^{N-1}\left[1-\frac{2 \sum_{k=1}^{K} \min \left(f_{k}(x, y), \hat{f}_{k}(x, y)\right)}{\sum_{k=1}^{K} f_{k}(x, y)+\hat{f}_{k}(x, y)}\right]
$$

This coefficient is also called as percentage similarity measures the similarity between different samples, communities and quadrates.

\subsection{Edge Based metrics}

\subsubsection{Laplacian Mean Square Error (LMSE)}

This measure is based on importance of edges measurement. The large value of Laplacian mean square error means that the image is poor quality. LMSE is defined as 
The International Journal of Multimedia \& Its Applications (IJMA) Vol.4, No.6, December 2012

$$
L M S E=\frac{\sum_{x=0}^{M-1} \sum_{y=0}^{N-1}[L(f(x, y))-L(\hat{f}(x, y))]^{2}}{\sum_{x=0}^{M-1} \sum_{y=0}^{N-1}[L(f(x, y))]^{2}}
$$

\subsection{HVS based metrics}

\subsubsection{Universal Image Quality Index (UQI)}

It is a measure used to find the image distortion. It is mathematically defined by making the image distortion relative to the reference image as a combination of three factors: Loss of correlation, Luminance distortion and contrast distortion.

If two images $f(x, y)$ and $\hat{f}(x, y)$ are considered as a matrices with $\mathrm{M}$ column and $\mathrm{N}$ rows containing pixel values $f(x, y)$ and $\hat{f}(x, y)$ respectively the universal image quality index $\mathrm{Q}$ may be calculated as a product of three components

$$
Q=\frac{\sigma_{\hat{f}}}{\sigma_{f} \sigma_{\hat{f}}} \cdot \frac{2 \bar{f} \overline{\hat{f}}}{f^{2}+\hat{f}^{2}} \cdot \frac{2 \sigma_{f} \sigma_{\hat{f}}}{\sigma_{f}^{2}+\sigma_{\hat{f}}^{2}}
$$

Where $\bar{f}=\frac{1}{M N} \sum_{x=0}^{M-1} \sum_{y=0}^{N-1} f(x, y) \quad$ and $\quad \overline{\hat{f}}=\frac{1}{M N} \sum_{x=0}^{M-1} \sum_{y=0}^{N-1} \hat{f}(x, y)$

$$
\begin{aligned}
& \sigma_{\hat{f f}}=\frac{1}{M+N-1} \sum_{x=0}^{M-1} \sum_{y=0}^{N-1}(f(x, y)-\bar{f})(\hat{f}(x, y)-\overline{\hat{f}}) \\
& \sigma_{f}^{2}=\frac{1}{M+N-1} \sum_{x=0}^{M-1} \sum_{y=0}^{N-1}(f(x, y)-\bar{f})^{2} \text { and } \sigma_{\hat{f}}^{2}=\frac{1}{M+N-1} \sum_{x=0}^{M-1} \sum_{y=0}^{N-1}(\hat{f}(x, y)-\overline{\hat{f}})^{2}
\end{aligned}
$$

The first component is the correlation coefficient which measures the degree of linear correlation between images. It varies in the range $[-1,1]$. The best value 1 is obtained when the images are linearly related. The second component measures how close the mean luminance is between images with a range $[0,1]$. The third component measures the contrasts of the images the value range for this component is $[0,1]$. The range of values for $\mathrm{Q}$ is $[-1,1]$. The best value 1 is achieved if and only if the images are identical.

4.4.2 Structural similarity (SSIM) index is a method for measuring the similarity between two images [28]. The SSIM index is a full reference metric, in other words, the measuring of image quality based on an initial uncompressed or distortion-free image as reference. SSIM is designed to improve on traditional methods like peak signal-to-noise ratio (PSNR) and mean squared error (MSE), which have proved to be inconsistent with human eye perception [10].

The SSIM metric is calculated on various windows of an image. The measure between two windows $x$ and $y$ of common size $N \times N$ is [28]:

$$
\operatorname{SSIM}(x, y)=\frac{\left(2 \mu_{x} \mu_{y}+c_{1}\right)\left(2 \sigma_{x y}+c_{2}\right)}{\left(\mu_{x}^{2}+\mu_{y}^{2}+c_{1}\right)\left(\sigma_{x}^{2}+\sigma_{y}^{2}+c_{2}\right)}
$$

Where

$\mu_{x}$ is the average of $x$ and $\mu_{y}$ is the average of $y$ 
The International Journal of Multimedia \& Its Applications (IJMA) Vol.4, No.6, December 2012

$>\sigma_{x}^{2}$ is the variance of $x$ and $\sigma_{y}^{2}$ is the variance of $y$

$>\sigma_{x y}$ is the covariance of $x$ and $y$

$>c_{1}=\left(k_{1} L\right)^{2}, c_{2}=\left(k_{2} L\right)^{2}$ two variables to stabilize the division with weak denominator

$>L$ is the dynamic range of the pixel values (typically this is $2^{\# \text { bits per pixel }}-1$ )

$>k_{1}=0.01$ and $k_{2}=0.03$ by default

In order to evaluate the image quality this formula is applied only on luma. The resultant SSIM index is a decimal value between -1 and 1 , and value 1 is only reachable in the case of two identical sets of data. Typically it is calculated on window sizes of $8 \times 8$. The window can be displaced pixel-by-pixel on the image.

\section{RESULTS}

The algorithms were applied to denoise the X-ray images contaminated with Poisson noise and the MR images contaminated with Rician noise. Three variance stabilization transforms square root, Freeman \& Tukey and Anscombe are used in combination with patch based algorithms Nonlocal means and BM3D. The quality metrics were tabulated as below. The denoised images of Poisson noise (Anscombe+NLM, Anscombe+BM3D) and of Rician noise (Square Root +NLM, Square root+BM3D, Freeman+NLM and Freeman+BM3D) are presented below.

Table 2: Performance evaluation of denoising algorithms for Poisson noise

\begin{tabular}{|l|l|l|l|l|l|l|l|l|}
\hline Poisson & \multicolumn{4}{|c|}{ NLM } & \multicolumn{4}{c|}{ BM3D } \\
\hline VST & $\begin{array}{l}\text { Square } \\
\text { root }\end{array}$ & $\begin{array}{l}\text { Freeman\& } \\
\text { Tukey }\end{array}$ & Anscombe & $\begin{array}{l}\text { Anscombe } \\
\text { Exact } \\
\text { Inverse }\end{array}$ & $\begin{array}{l}\text { Square } \\
\text { root } \\
\text { e }\end{array}$ & $\begin{array}{l}\text { Freeman } \\
\text { Tukey }\end{array}$ & $\begin{array}{l}\text { Anscombe } \\
\text { Exact } \\
\text { Inverse }\end{array}$ \\
\hline MSE & 65.5528 & 15.5524 & 15.3016 & 15.3002 & 61.5528 & 13.5524 & 13.3016 & 13.3002 \\
\hline SNR & 22.8880 & 32.4486 & 32.5369 & 32.5373 & 27.8880 & 34.4486 & 34.5369 & 34.5373 \\
\hline RMSE & 8.8456 & 4.6814 & 4.6471 & 4.6469 & 7.8456 & 3.6814 & 3.6471 & 3.6469 \\
\hline PSNR & 28.2486 & 35.8209 & 34.9021 & 35.9025 & 33.2486 & 39.8209 & 39.9021 & 39.9025 \\
\hline ME & 10.3824 & 4.9972 & 4.9541 & 4.9541 & 9.3824 & 4.3972 & 4.3541 & 4.3541 \\
\hline ME4 & 10.9798 & 5.6085 & 5.6618 & 5.6618 & 10.7798 & 5.1085 & 5.0618 & 5.0618 \\
\hline UQI & 0.3976 & 0.6197 & 0.6005 & 0.6115 & 0.4976 & 0.6797 & 0.6805 & 0.6815 \\
\hline SSIM & 0.5726 & 0.8973 & 0.8973 & 0.8974 & 0.6726 & 0.9173 & 0.9173 & 0.9174 \\
\hline AD & 0.6749 & 0.4291 & 0.3765 & 0.3841 & 0.4749 & 0.6291 & 0.3765 & 0.3841 \\
\hline SC & 1.0082 & 1.0137 & 1.0104 & 1.0104 & 1.0082 & 1.0137 & 1.0104 & 1.0104 \\
\hline NK & 0.9943 & 0.9929 & 0.9945 & 0.9945 & 0.9943 & 0.9929 & 0.9945 & 0.9945 \\
\hline MD & 58.0579 & 42.3644 & 40.5074 & 39.8074 & 53.0579 & 40.3644 & 39.7074 & 39.7074 \\
\hline LMSE & 8.7992 & 0.6107 & 0.6108 & 0.6108 & 7.7992 & 0.5107 & 0.5108 & 0.5108 \\
\hline NAE & 0.0484 & 0.0227 & 0.0226 & 0.0225 & 0.0484 & 0.0227 & 0.0226 & 0.0225 \\
\hline
\end{tabular}


The International Journal of Multimedia \& Its Applications (IJMA) Vol.4, No.6, December 2012

Table 3: Performance evaluation of denoising algorithms for Rician noise

\begin{tabular}{|l|l|l|l|l|l|l|l|l|}
\hline Rician & \multicolumn{4}{|c|}{ NLM } & \multicolumn{4}{c|}{ BM3D } \\
\hline VST & $\begin{array}{l}\text { Square } \\
\text { root }\end{array}$ & $\begin{array}{l}\text { Freeman\& } \\
\text { Tukey }\end{array}$ & Anscombe & $\begin{array}{l}\text { Anscombe } \\
\text { Exact } \\
\text { Inverse }\end{array}$ & $\begin{array}{l}\text { Square } \\
\text { root }\end{array}$ & $\begin{array}{l}\text { Freeman\& } \\
\text { Tukey }\end{array}$ & $\begin{array}{l}\text { Anscombe } \\
\text { Exact } \\
\text { Inverse }\end{array}$ \\
\hline MSE & 258.6277 & 171.6379 & 176.5006 & 172.0002 & 238.3277 & 161.5379 & 164.8006 & 164.8012 \\
\hline SNR & 16.1326 & 18.6727 & 17.9969 & 19.0012 & 18.1236 & 19.7737 & 19.6969 & 19.6969 \\
\hline RMSE & 17.4379 & 13.0098 & 13.8375 & 13.5375 & 15.4379 & 12.7098 & 12.8375 & 12.8375 \\
\hline PSNR & 24.3694 & 28.1544 & 28.1615 & 28.2515 & 27.3694 & 29.0584 & 28.9715 & 28.9715 \\
\hline ME & 18.3813 & 15.6106 & 15.6515 & 15.3515 & 17.8313 & 14.8106 & 14.9515 & 14.9515 \\
\hline ME4 & 20.1132 & 16.7326 & 16.8840 & 16.3840 & 19.9132 & 16.6336 & 16.7840 & 16.7840 \\
\hline UQI & 0.3880 & 0.5015 & 0.5020 & 0.5170 & 0.4880 & 0.5625 & 0.5620 & 0.5620 \\
\hline SSIM & 0.4843 & 0.5980 & 0.5991 & 0.6012 & 0.5143 & 0.6180 & 0.6167 & 0.6167 \\
\hline AD & -7.2958 & -6.7430 & -6.6995 & -6.7855 & -6.2958 & -5.9450 & -6.1955 & -6.1955 \\
\hline SC & 0.8489 & 0.9056 & 0.9016 & 0.9062 & 0.9489 & 0.9656 & 0.9612 & 0.9612 \\
\hline NK & 1.0111 & 1.0071 & 1.0092 & 1.0092 & 1.0111 & 1.0071 & 1.0092 & 1.0092 \\
\hline MD & 81.3348 & 59.4538 & 59.7788 & 59.1788 & 71.3948 & 58.0538 & 58.0788 & 58.0788 \\
\hline LMSE & 5.0087 & 2.3968 & 2.8241 & 2.5861 & 4.1097 & 1.8398 & 1.8461 & 1.8461 \\
\hline NAE & 0.2035 & 0.1771 & 0.1787 & 0.1787 & 0.1935 & 0.1571 & 0.1587 & 0.1587 \\
\hline
\end{tabular}
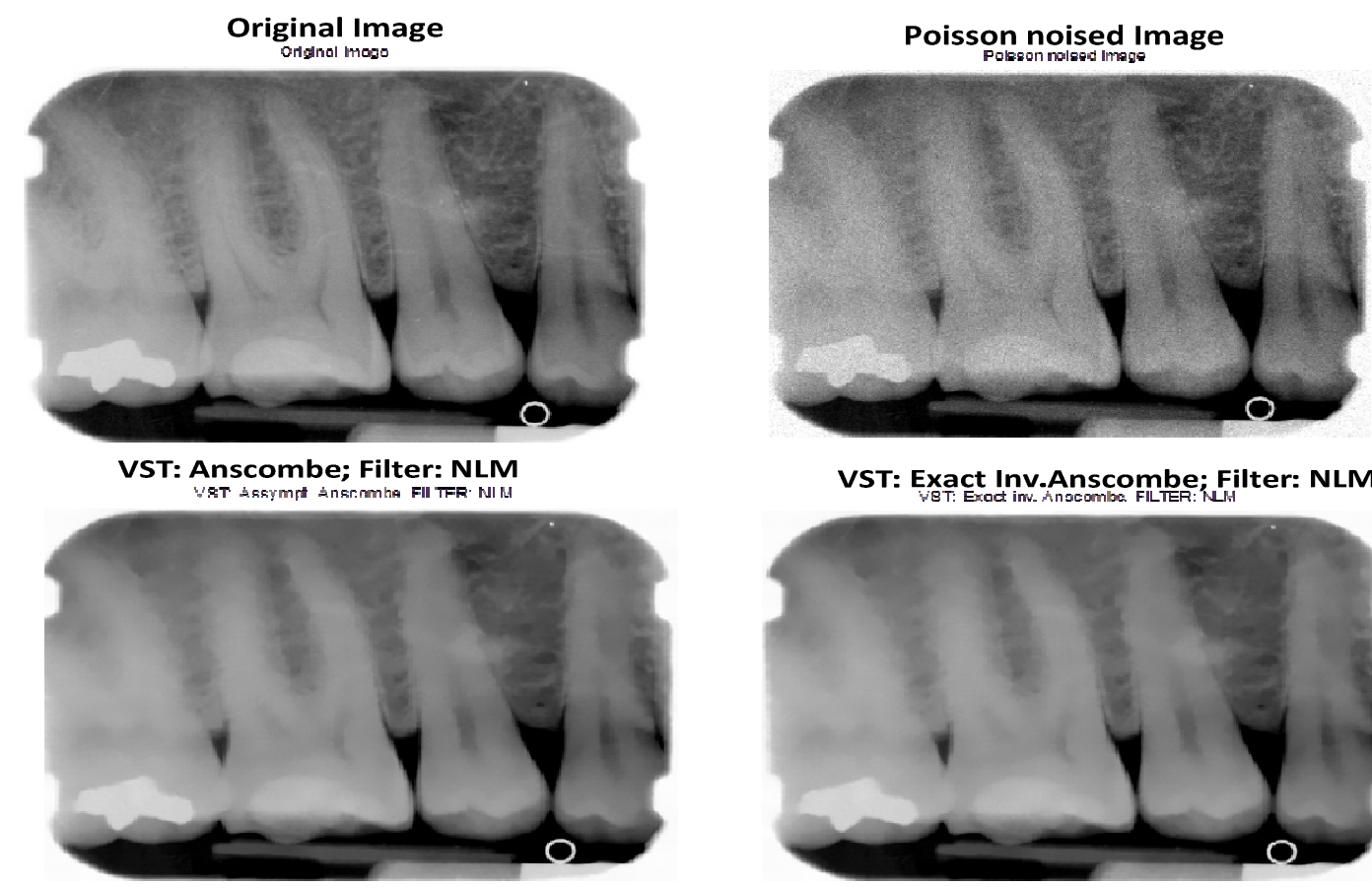

VST: Exact Inv.Anscombe; Filter: NLM

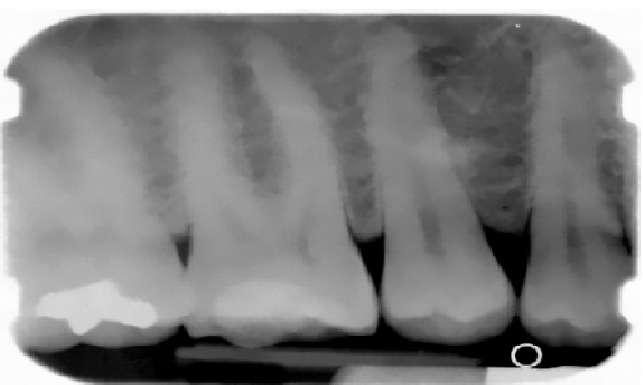

VST: Anscobe, Filter:BM3D
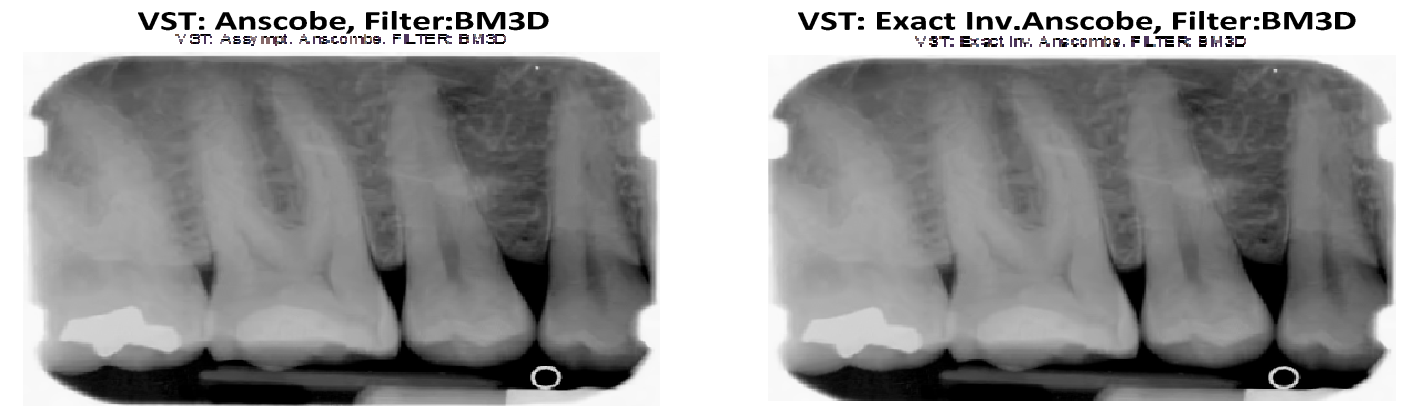
The International Journal of Multimedia \& Its Applications (IJMA) Vol.4, No.6, December 2012
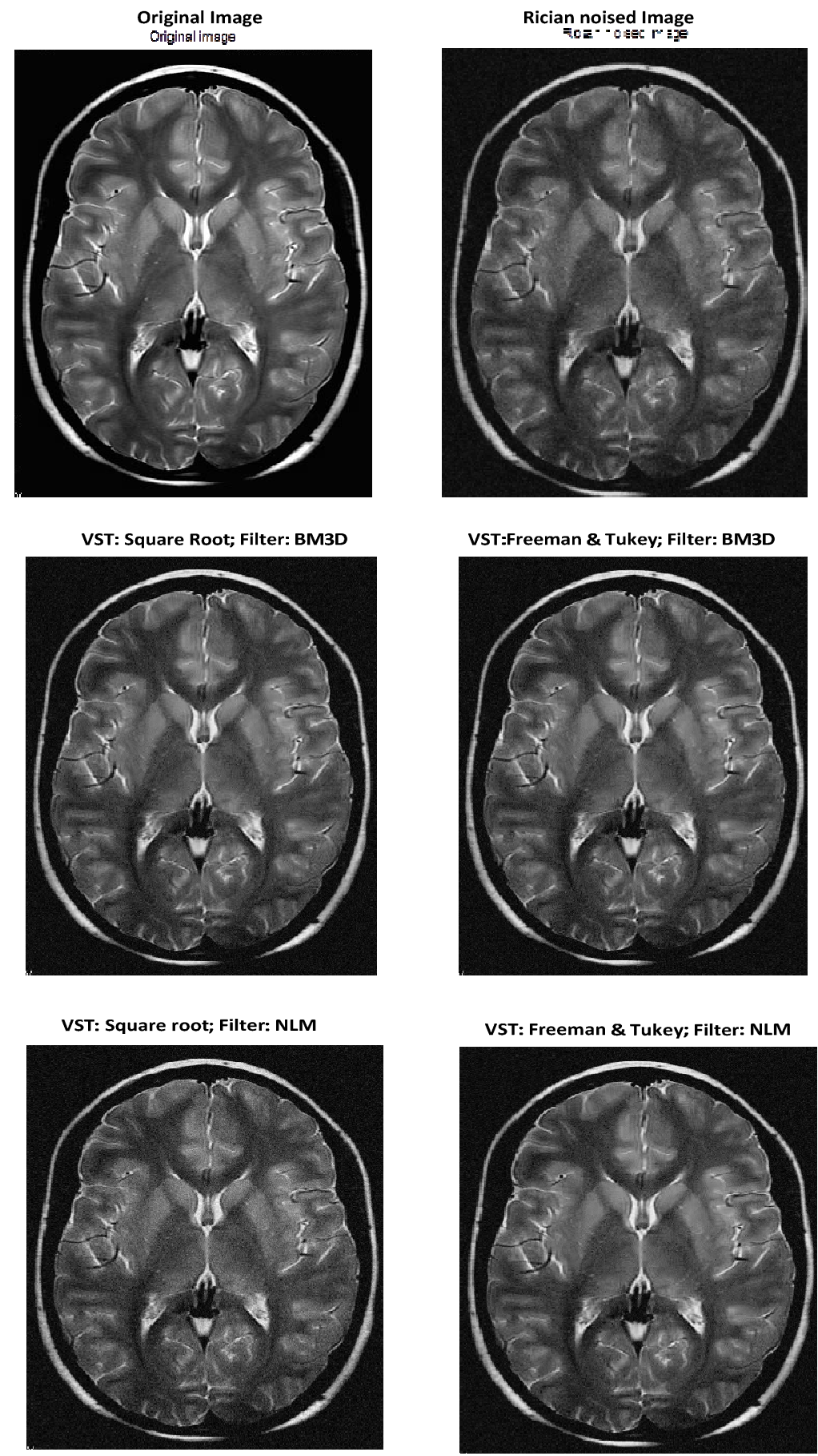

\section{CONCLUSIONS AND FUTURE WORK}

The development and implementation of denoising filters using variance stabilization transform and patch based algorithms were carried and evaluated their performance using various image quality metrics. The developed algorithms are applied on the x-ray and MR Images and the visual appearance after denoising was improved, the contrast of the images are also improved. Further 
The International Journal of Multimedia \& Its Applications (IJMA) Vol.4, No.6, December 2012

enhancement can be done using the wavelet frames such as complex dual tree wavelet transforms and double density wavelet transforms etc.

\section{REFERENCES}

[1] J.W. Goodman, "Some fundamental properties of speckle," J. Opt. Soc. Am., vol. 66, no. 11, pp. 1145-1149, 1976.

[2] C.B. Burckhardt, "Speckle in ultrasound B-mode scans," IEEE Trans. Sonics Ultrasonics, vol. SU-25, no. 1 , pp. 1-6, 1978.

[3] Z. Tao, H. D. Tagare, and J. D. Beaty. Evaluation of four probability distribution models for speckle in clinical cardiac ultrasound images. IEEE Transactions on Medical Imaging, 25(11):1483\{1491, 2006.

[4] P. C. Tay, S. T. Acton, and J. A. Hossack. A stochastic approach to ultrasound despeckling. In Biomedical Imaging: Nano to Macro, 2006. 3rd IEEE International Symposium on, pages 221\{224, 2006.

[5] J.S. Lee, "Digital image enhancement and noise filtering by using local statistics," IEEE Trans. Pattern Anal. Mach. Intell., PAMI-2, no. 2, pp. 165-168, 1980.

[6] J.S. Lee, "Speckle analysis and smoothing of synthetic aperture radar images," Comp. Graphics Image Process., vol. 17, pp. 24-32, 1981, doi:10.1016/S0146-664X(81)80005-6.

[7] J.S. Lee, "Refined filtering of image noise using local statistics," Comput. Graphics Image Process, vol. 15 , pp. 380-389, 1981.

[8] V.S. Frost, J.A. Stiles, K.S. Shanmungan, and J.C. Holtzman, "A model for radar images and its application for adaptive digital filtering of multiplicative noise," IEEE Trans. Pattern Anal. Mach. Intell., vol. 4, no. 2, pp. 157-165, 1982.

[9] D.T. Kuan and A.A. Sawchuk, "Adaptive noise smoothing filter for images with signal dependent noise," IEEE Trans. Pattern Anal. Mach. Intell., vol. PAMI-7, no. 2, pp. 165-177, 1985.

[10] D.T. Kuan, A.A. Sawchuk, T.C. Strand, and P. Chavel, "Adaptive restoration of images with speckle,” IEEE Trans. Acoust., vol. ASSP-35, pp. 373-383, 1987, doi:10.1109/TASSP.1987.1165131.

[11] J. Saniie, T. Wang, and N. Bilgutay, "Analysis of homomorphic processing for ultrasonic grain signal characterization," IEEE Trans. Ultrason. Ferroelectr. Freq. Control, vol. 3, pp. 365-375, 1989, doi:10.1109/58.19177.

[12] A. Pizurica, A. M.Wink, E. Vansteenkiste, W. Philips, and J. Roerdink, "A review of wavelet denoising in mri and ultrasound brain imaging," Curr. Med. Imag. Rev., vol. 2, no. 2, pp. 247-260, 2006.

[13] D.L. Donoho, "Denoising by soft thresholding," IEEE Trans. Inform. Theory, vol. 41, pp. 613-627, 1995.

[14] X. Zong, A. Laine, and E. Geiser, "Speckle reduction and contrast enhancement of echocardiograms via multiscale nonlinear processing," IEEE Trans. Med. Imaging, vol. 17, no. 4, pp. 532-540, 1998.

[15] X. Hao, S. Gao, and X. Gao, "A novel multiscale nonlinear thresholding method for ultrasonic speckle suppressing,” IEEE Trans. Med. Imaging, vol. 18, no. 9, pp. 787-794, 1999.

[16] F.N.S Medeiros, N.D.A. Mascarenhas, R.C.P Marques, and C.M. Laprano, "Edge preserving wavelet speckle filtering," in 5th IEEE Southwest Symposium on Image Analysis and Interpretation, Santa Fe, NM, pp. 281-285, April 7-9, 2002, doi:10.1109/IAI.2002.999933.

[17] C. M. Sehgal, "Quantitative relationship between tissue composition and scattering of ultrasound", J.Acoust. Soc. Am., vol. 94, No.3, pp.1944-1952, Oct.1993.

[18] J. T. M. Verhoeven and J. M. Thijssen, "Improvement of lesion detectability by speckle reduction filtering: A quantitative study", Ultrason. Imag., vol. 15, pp.181-204, 1993.

[19] Paul Butler, “Applied Radiological Imaging for Medical Students", I ${ }^{\text {st }}$ Edition, Cambridge University Press, 2007.

[20] Rangaraj M. Rangayyan, "Biomedical Signal Analysis A Case study Approach”, IEEE Press, 2005.

[21] Stephane Mallat, "A Wavelet Tour of signal Processing", Elsevier, 2006.

[22] D L Donoho and M. Jhonstone, "Wavelet shrinkage: Asymptopia?", J.Roy.Stat.Soc., SerB, Vol.57, pp. 301-369, 1995.

[23] D L Donoho, "De-Noising by Soft-Thresholding", IEEE Transactions on Information Theory, vol.41, No.3, May 1995. 
The International Journal of Multimedia \& Its Applications (IJMA) Vol.4, No.6, December 2012

[24] David L. Donoho and Iain M. Johnstone.,"Adapting to unknown smoothness via wavelet shrinkage”, Journal of the American Statistical Association, vol.90, no432, pp.1200-1224, December 1995. National Laboratory, July 27, 2001.

[25] R. Coifman and D. Donoho, "Translation invariant de-noising," in Lecture Notes in Statistics: Wavelets and Statistics, vol. New York: Springer-Verlag, pp. 125--150, 1995.

[26] S. G. Mallat and W. L. Hwang, "Singularity detection and processing with wavelets," IEEE Trans. Inform. Theory, vol. 38, pp. 617-643, Mar. 1992.

[27] I.Daubechies, "Ten Lectures on Wavelets”, SIAM Publishers, 1992.

[28] Z. Wang, A. C. Bovik, H. R. Sheikh and E. P. Simoncelli, "Image quality assessment: From error visibility to structural similarity," IEEE Transactions on Image Processing, vol. 13, no. 4, pp. 600-612, Apr. 2004.

[29] Antoni Buades, Bartomeu Coll, Jean-Michel Morel, Non-Local Means Denoising, Image Processing On Line, 2011

[30] Kostadin Dabov, Alessandro Foi, Vladimir Katkovnik, Karen EgiaZarian, "Image denoising by sparse 3D transform domain collaborative filtering", IEEE Trans. On Image Processing, Vol.16, No.8, August 2007.

[31] N. G. Kingsbury, "The dual-tree complex wavelet transform: A new technique for shift invariance and directional filters," in Proc. Eighth IEEE DSP Workshop, Salt Lake City, UT, Aug. 9-12, 1998.

[32] I.W.Selesnick, “The double density dual tree DWT”, IEEE Transactions on Signal Processing, Vol 52. No.5, May 2004. 